\title{
The Cost-Effectiveness of Screening Men Who Have Sex With Men for Rectal Chlamydial and Gonococcal Infection to Prevent HIV Infection
}

\author{
Harrell W. Chesson, PhD*, Kyle T. Bernstein, $\mathrm{PhD}^{\dagger}$, Thomas L. Gift, PhD ${ }^{\star}$, Julia L. Marcus, \\ $\mathrm{MPH}^{\dagger}$, Sharon Pipkin, $\mathrm{MPH}^{\dagger}$, Charlotte K. Kent, $\mathrm{PhD}^{*}$ \\ ${ }^{*}$ Centers for Disease Control and Prevention, Atlanta, GA \\ †San Francisco Department of Public Health, San Francisco, CA
}

\begin{abstract}
Background: Men who have sex with men (MSM) who have a current or recent history of rectal Chlamydia trachomatis (CT) and Neisseria gonorrhoeae $(G C)$ infection are at greater risk for HIV than MSM with no history of rectal infection. Screening and treating MSM for rectal $C T / G C$ infection may help reduce any increased biological susceptibility to HIV infection.
\end{abstract}

Methods: We used 2 versions of a Markov state-transition model to examine the impact and costeffectiveness of screening MSM for rectal $C T / G C$ infection in San Francisco: a static version that included only the benefits to those screened and a dynamic version that accounted for populationlevel impacts of screening. HIV prevention through reduced susceptibility to HIV was the only potential benefit of rectal $C T / G C$ screening that we included in our analysis. Parameter values were based on San Francisco program data and the literature.

Results: In the base case, the cost per quality-adjusted life year gained through screening MSM for rectal $C T / G C$ infection was $\$ 16,300$ in the static version of the model. In the dynamic model, the cost per quality-adjusted life year gained was less than $\$ 0$, meaning that rectal screening was cost-saving. The impact of rectal $C T / G C$ infection on the risk of HIV acquisition was the most influential model parameter.

Conclusions: Although more information is needed regarding the impact of rectal $C T / G C$ screening on HIV incidence, rectal $C T / G C$ screening of MSM can potentially be a cost-effective, scalable intervention targeted to at-risk MSM in certain urban settings such as San Francisco.

\begin{abstract}
Men who have sex with men (MSM) who have a current or recent history of rectal Chlamydia trachomatis (CT) and/ or Neisseria gonorrhoeae $(G C)$ infection are at greater risk for HIV than MSM with no history of rectal infection. ${ }^{1-3}$ This increased risk may be attributable to biological factors, behavioral factors, or both. ${ }^{1-3}$ Screening and treating MSM for rectal $C T$ and $G C$ infection may help reduce any increased biological susceptibility to HIV infection and identify men at increased risk for HIV infection. ${ }^{1-3}$
\end{abstract}

Correspondence: Harrell W. Chesson, PhD, CDC Mailstop E-80, 1600 Clifton Rd, Atlanta, GA 30333. hbc7@cdc.gov. Supplemental digital content is available for this article. Direct URL citations appear in the printed text, and links to the digital files are provided in the HTML text of this article on the journal's Web site (http://www.stdjournal.com). 
The burden of rectal sexually transmitted diseases (STDs) among MSM has been well documented. ${ }^{4-10}$ However, most infections are asymptomatic, suggesting that routine screening is needed to identify and treat rectal STDs. ${ }^{6,10}$ Although the Centers for Disease Control and Prevention recommends rectal screening for $C T$ and $G C$ infection among men who have had receptive anal intercourse in the past year, ${ }^{11}$ rectal screening rates are limited. For example, most MSM with HIV in urban areas are not screened for rectal $C T$ or $G C$ infection in any given year, although screening rates can vary from clinic to clinic. ${ }^{12} \mathrm{San}$ Francisco is one example of a setting with relatively high rectal screening rates because the San Francisco Department of Health (SFDPH) has supported extragenital testing in a variety of clinical sites. The purpose of this study was to estimate the impact and cost-effectiveness of screening for rectal $C T$ and $G C$ infection among MSM in San Francisco, using estimates of current rectal screening coverage rates.

\section{MATERIALS AND METHODS}

\section{The Model}

We used a Markov state-transition model to examine the potential impact of screening MSM for rectal $C T$ and $G C$ infection. The model consists of 4 mutually exclusive health states based on HIV infection status and rectal $C T / G C$ infection status (Fig. 1), where $C T / G C$ denotes $C T$ and/or $G C$ infection. Transition from one state to another occurs when rectal $C T / G C$ infection is acquired, when rectal $C T / G C$ infection is cleared, or when HIV is acquired. The incidence rate of rectal $C T / G C$ infection is $\lambda_{\mathrm{STD}} \Omega_{\mathrm{STD}}$ and $\hat{\lambda}_{\mathrm{STD}} \Omega_{\mathrm{STD}}$ among those without and with HIV, respectively, where $\lambda_{\mathrm{STD}}$ and $\hat{\lambda}_{\mathrm{STD}}$ are the incidence rates of rectal $G C$ infection at the onset of the screening program among those without and with $\mathrm{HIV}$, respectively, and $\Omega_{\mathrm{STD}}$ is an adjustment factor to account for changes in the prevalence of $C T / G C$ in sex partners over time as a result of the screening program. The clearance rate $(r)$ of rectal $C T / G C$ infection is a function of 2 factors: duration of infection and screening rates. The HIV incidence rate among those without rectal $C T / G C$ infection is $\lambda_{\mathrm{HIV}} \Omega_{\mathrm{HIV}}$, where $\lambda_{\mathrm{HIV}}$ is the HIV incidence at the onset of the screening program and $\Omega_{\mathrm{HIV}}$ is an adjustment factor to account for changes in the prevalence of HIV in sex partners over time. The rate of HIV incidence among those with rectal $C T / G C$ infection was assumed to be $\theta$ times that of those without rectal $C T / G C$ infection. The population of MSM was assumed to be 65,000 , with an initial HIV prevalence of $25 \% .{ }^{13}$ Initially, 1511 of the 48,750 MSM without HIV and 1576 of the 16,250 MSM with HIV were assumed to have rectal $C T / G C$ infection. We performed all calculations using Excel 2007 (Microsoft Corporation, Redmond, WA). A more detailed description of the model is provided in a supplemental appendix available from the lead author upon request (http://links.lww.com/OLQ/A58).

\section{Parameter Values}

Parameter values and costs applied in the model were based on the literature and on data from SFDPH, as listed in Table 1. Data from SFDPH were used to estimate annual incidence rates of rectal $C T / G C$ infection in MSM with and without HIV (0.129 and 0.041, respectively, as described in Table 1). HIV incidence among MSM without rectal $C T / G C$ infection was based on HIV incidence rates reported by SFDPH and additional assumptions 
(as described in Table 1). ${ }^{13}$ The relative risk $\theta$ of acquiring HIV among those with rectal $C T / G C$ infection was 1.9 (range, 1.2-2.6), based on HIV incidence reported in a retrospective cohort analysis of HIV-uninfected MSM diagnosed as having rectal $C T / G C$ infection, ${ }^{1}$ along with the calculation of HIV incidence among MSM without rectal $C T / G C$ (Table 1).

Duration of rectal $C T / G C$ infection in the absence of screening was assumed to be 9 months (range, 6 months-1 year). ${ }^{14,15}$ The annual rates of rectal $C T / G C$ screening was assumed to be 0.37 (range, 0.15-0.65) in MSM without HIV and 0.58 (range, 0.25-1) in MSM with HIV (Table 1).

The San Francisco City Clinic uses Gen-Probe APTIMA Combo 2 for testing rectal specimens, ${ }^{1}$ for which sensitivities and specificities of $92.3 \%$ and $98.7 \%$, respectively, have been reported for rectal $G C(93.5 \%$ and $97.7 \%$, respectively, for rectal $C T) .{ }^{16}$ For simplicity, we assumed $100 \%$ sensitivity and specificity of screening. Treatment has been documented in more than $95 \%$ of those with rectal $C T / G C$ in San Francisco, with a median time to treatment of 2 days (SFDPH, unpublished data). Given this high rate of treatment, we assumed for simplicity that all rectal $C T / G C$ infections detected through screening would be treated successfully immediately upon detection.

We estimated 2 model versions: a static version and a dynamic version. The static version included benefits of rectal $C T / G C$ screening only to those who are screened, whereas the dynamic version included benefits of rectal $C T / G C$ screening to those who are screened, their partners, their partners' partners, and so on. In the dynamic version, the adjustment factor $\left(\Omega_{\mathrm{STD}}\right)$ was used to account for changes in the prevalence of $C T / G C$ infection in sex partners over time as a result of rectal $C T / G C$ screening. The adjustment factor in week $t+1$ was calculated based on the ratio of rectal $C T / G C$ prevalence in week $t$ in the scenario of rectal screening to rectal $C T / G C$ prevalence in week $t$ in the scenario of no-rectal screening, raised to the power of 0.5 . The ratio was raised to the power of 0.5 because rectal $C T / G C$ screening was assumed to have less of an impact on genital $C T / G C$ prevalence than on rectal $C T / G C$ prevalence, similar to the way in which female-only STD screening might have less of an impact on male STD prevalence than female STD prevalence. ${ }^{17}$ The adjustment factor $\left(\Omega_{\mathrm{HIV}}\right)$ for changes in the prevalence of HIV in sex partners was calculated in an analogous manner as the adjustment factor for $C T / G C\left(\Omega_{\mathrm{STD}}\right)$. The adjustment factors $\left(\Omega_{\mathrm{HIV}}\right.$ and $\left.\Omega_{\mathrm{STD}}\right)$ were varied only in the dynamic version of the model; in the static model, both values were set equal to 1 and held constant.

The number of quality-adjusted life years (QALYs) lost per HIV infection, the direct costs for testing and treatment for $C T / G C$ infection, and the direct lifetime medical cost per case of HIV were drawn from the literature. ${ }^{18-23}$ All costs were updated to 2011 US dollars using the medical care component of the consumer price index (www.bls.gov/cpi/data.htm).

\section{Cost-Effectiveness}

The study question we addressed is as follows: what is the cost-effectiveness of current screening and treatment for rectal $C T / G C$ infection among MSM in San Francisco compared with a strategy of no screening? HIV prevention was the only benefit of rectal $C T / G C$ 
screening that we assessed; we did not include other health and economic benefits of treating rectal $C T / G C$ infection. Costs and benefits were assessed from the health system perspective; we did not include nonmedical costs such as patient time and transportation costs or indirect costs (lost productivity) of HIV or rectal STDs. All future costs and benefits were discounted at 3\% annually. We applied a 10-year time frame and a lifetime analytic horizon; that is, we included all program costs over the 10-year time frame (STD screening and treatment costs) as well as the lifetime costs and lifetime number of QALYs lost for the HIV cases that occurred over the 10-year time frame.

The cost-effectiveness of screening was expressed in terms of cost per QALY gained and calculated as the incremental cost of $C T / G C$ screening divided by the incremental number of QALYs gained by $C T / G C$ screening. The incremental cost of $C T / G C$ screening was calculated as the total costs of $C T / G C$ screening (cost of screening for both $C T$ and $G C$, cost of treating $C T, G C$, or both; and HIV costs) minus the HIV costs in the scenario of no$C T / G C$ screening. The incremental number of QALYs gained by $C T / G C$ screening was calculated as the lifetime number of QALYs lost due to HIV in the scenario of no- $C T / G C$ screening minus the lifetime number of QALYs lost due to HIV in the screening scenario.

\section{Sensitivity Analysis}

In sensitivity analyses, we varied the parameter values according to the ranges described in Table 1 to see how the estimated cost per QALY gained by rectal $C T / G C$ screening would change. We first conducted 1-way sensitivity analyses, calculating the cost per QALY gained by rectal $C T / G C$ screening when varying one parameter at a time from its lower bound to its upper bound value while holding all other parameters at their base case values. The parameters varied in the 1-way sensitivity analyses were as follows: the incidence of rectal $C T / G C$ infection ( $\lambda_{\mathrm{STD}}$ and $\hat{\lambda}_{\mathrm{STD}}$ ), HIV incidence among those without rectal $C T / G C$ infection ( $\lambda_{\text {HIV }}$ ), the relative risk of HIV among those with rectal $C T / G C$ infection $(\theta)$, the duration of $C T / G C$ infection (d), the lifetime cost and number of QALYs lost per HIV case, the cost of rectal $C T / G C$ screening and treatment, and the rate of rectal $C T / G C$ screening. We then conducted probabilistic sensitivity analyses in which all of these parameter values (except the rate of $C T / G C$ of screening) were varied simultaneously, assuming a uniform distribution for each parameter between its lower and upper bound values. Finally, we conducted a threshold analysis to determine what values of $\theta$ (the relative risk of acquiring HIV among those with rectal $C T / G C$ infection) would result in cost-effectiveness ratios of $\$ 25,000, \$ 50,000$, and $\$ 100,000$ per QALY gained by rectal $C T / G C$ screening.

\section{RESULTS}

In the base case, the cost per QALY gained by screening MSM for rectal $C T / G C$ infection was $\$ 16,300$ in the static version of the model and less than $\$ 0$ in the dynamic version of the model (Table 2). Thus, when taking into account dynamic reductions in $C T / G C$ prevalence among MSM over time as a result of rectal $C T / G C$ screening, the cost per QALY gained was less than $\$ 0$, meaning that rectal screening is cost-saving. In 1-way sensitivity analyses, the estimated cost per QALY gained by screening ranged from less than $\$ 0$ to $\$ 227,800$ in the static version of the model and from less than $\$ 0$ to $\$ 98,400$ in the dynamic version of the 
model (Table 3). The 2 most influential parameters were the relative risk of acquiring HIV among those with rectal $C T / G C$ infection and the duration of rectal $C T / G C$ infection. In the 1-way sensitivity analyses, the cost per QALY gained did not exceed $\$ 50,000$ in the static version of the model or $\$ 5000$ in the dynamic version of the model, except when varying the relative risk of $\mathrm{HIV}$ or the duration of rectal $C T / G C$ infection.

In the probabilistic sensitivity analyses, the cost per QALY gained by rectal $C T / G C$ screening ranged from less than $\$ 0$ to $\$ 403,100$ in $90 \%$ of the simulations using the static version of the model and from less than $\$ 0$ to $\$ 120,100$ in $90 \%$ of the simulations using the dynamic version of the model (Table 4). In the static version of the model, the cost per QALY was less than $\$ 0$ (cost-saving) in 31\% of the simulations and was less than $\$ 100,000$ in $75 \%$ of the simulations. In the dynamic version of the model, the cost per QALY was less than $\$ 0$ (cost-saving) in 59\% of the simulations and was less than $\$ 100,000$ in $94 \%$ of the simulations.

In threshold analyses of the relative risk of acquiring HIV among those with rectal $C T / G C$ infection (not shown), cost per QALY estimates of $\$ 25,000, \$ 50,000$, and $\$ 100,000$ were obtained when the relative risk was set to $1.80,1.60$, and 1.40, respectively, in the static version of the model and when the relative risk was set to $1.43,1.33$, and 1.20 , respectively, in the dynamic version of the model.

\section{DISCUSSION}

Our results suggest that screening MSM for rectal $C T / G C$ infection can be a cost-effective intervention to reduce HIV infection, particularly when taking into account the populationlevel reductions in $C T / G C$ prevalence in MSM over time as a result of screening and treatment. In many scenarios we examined, screening MSM for rectal $C T / G C$ infection was cost-saving, meaning that the discounted costs of screening and treatment were less than the discounted cost of averted HIV infections. Although there is no official cost-per-QALY cutoff to determine whether public health interventions in the United States are costeffective, a threshold of $\$ 50,000$ per QALY is often cited (a QALY can be thought of as 1 year of life in perfect health). ${ }^{24,25}$ In our sensitivity analyses, the cost per QALY gained by rectal screening was less than $\$ 50,000$ in $60 \%$ of the simulations when using the static version of the model and in $85 \%$ of the simulations when using the dynamic version of the model. Our base-case estimate of the cost per QALY gained by screening MSM for rectal $C T / G C$ ( $\$ 16,300$ in the static version of the model) is consistent with estimates of the cost per QALY gained by screening women and high-risk heterosexual men for urogenital $C T$ infection 26,27 and compares favorably with many clinical preventive services and public health interventions. ${ }^{28}$

The degree to which rectal $C T / G C$ increases susceptibility to HIV is the most important input in our model. The cost per QALY gained by screening MSM for rectal $C T / G C$ was less than $\$ 25,000$ in the dynamic version of our model when the relative risk of acquiring HIV among MSM with rectal $C T / G C$ infection (compared with MSM without rectal $C T / G C$ infection) was at least 1.43 . Thus, if rectal $C T / G C$ infection increases susceptibility to HIV by at least $43 \%$, then screening MSM for rectal $C T / G C$ infection would be a highly cost- 
effective strategy for HIV prevention, according to our dynamic model results. Such an increase in susceptibility seems plausible, given that a case-control study found an odds ratio of 4.73 (95\% confidence interval, 1.75-12.76) for HIV seroconversion among MSM with rectal gonorrhea compared with MSM controls. ${ }^{2}$ Furthermore, among MSM with a current rectal $C T / G C$ infection, HIV seroconversion was 8 times more likely among MSM with 2 prior $C T / G C$ infections than among MSM without a prior $C T / G C$ infection. ${ }^{1}$ In addition, there is a substantial overall body of evidence suggesting that STDs can facilitate the acquisition and transmission of HIV. ${ }^{29-34}$ However, it is difficult to determine how much of the increased risk of HIV in those with rectal $C T / G C$ infection is attributable to biological factors and how much is attributable to other factors such as sexual behaviors and sex partner characteristics. ${ }^{3,33,34}$ Future research is needed to help determine more precisely the causal role of rectal $C T / G C$ infection on HIV acquisition. ${ }^{1,3}$

Our study is subject to several important limitations. First, data are limited regarding the 2 most important parameters in our analysis: the relative risk of acquiring HIV among those with rectal $C T / G C$ infection (vs. those without) and the duration of rectal $C T / G C$ infection. To address this limitation, we conducted sensitivity analyses to show how the results can change when these key assumptions are varied. Second, we did not account specifically for repeat rectal $C T / G C$ infections. Evidence suggests that the risk of HIV seroconversion is higher for MSM with 1 and 2 repeat rectal $C T / G C$ infections than for MSM with a single rectal $C T / G C$ infection. ${ }^{1}$ Third, we used a relatively simple approach to estimate the impact of a rectal $C T / G C$ screening program. Given that the cost per QALY gained by rectal $C T / G C$ screening was notably lower in the dynamic version of our model than in the static version, future studies could use a more complex dynamic transmission model to examine the potential impact of screening in more detail. Fourth, we made several simplifying assumptions such as $100 \%$ sensitivity and specificity of screening and that all rectal $C T / G C$ infections detected would be treated successfully immediately upon detection. Although these optimistic assumptions can result in cost-effectiveness estimates that are unduly favorable to rectal screening, our analysis was otherwise conservative in that HIV prevention through reduced susceptibility to HIV was the only potential benefit of rectal $C T / G C$ screening that we included. For example, we did not consider the possibility that rectal $C T / G C$ screening in MSM with HIV could reduce the probability of HIV transmission to their sex partners. Similarly, we focused solely on biological impacts of rectal $C T / G C$ infection on HIV acquisition, although it is possible that rectal $C T / G C$ screening could reduce the risk of HIV acquisition and transmission through behavioral impacts, as well. Rectal $C T / G C$ screening could be markedly more cost-effective than we estimated, had we included all of these potential benefits of screening. Fifth, we assumed that our model inputs would be constant over time. For example, we did not allow for the possibility that increased use of antiretroviral therapy might affect the average cost per case of HIV, the average impact of HIV on length and quality of life, and the HIV incidence rate. Finally, our model used parameter values specific to MSM in San Francisco, and our results might not be generalizable to other areas.

Despite limitations, our model offers a useful approximation of the health impact and costeffectiveness of rectal $C T / G C$ screening in San Francisco to reduce future HIV acquisition. If rectal $C T / G C$ infection does indeed increase susceptibility to HIV, rectal $C T / G C$ screening 
of MSM could be a cost-effective tool to prevent HIV and might even pay for itself in terms of averted HIV treatment costs. The Centers for Disease Control and Prevention has called for a high-impact HIV prevention approach, defined as the use of combinations of "scientifically proven, cost-effective, and scalable interventions targeted to the right populations in the right geographic areas." 35 Although more information is needed regarding the impact of rectal $C T / G C$ screening on the risk of HIV acquisition, our analysis shows that rectal $C T / G C$ screening of MSM can potentially be a cost-effective, scalable intervention targeted to at-risk MSM in certain urban settings such as San Francisco.

\section{Supplementary Material}

Refer to Web version on PubMed Central for supplementary material.

\section{Acknowledgments}

The findings and conclusions in this report are those of the authors and do not necessarily represent the views of the Centers for Disease Control and Prevention.

\section{REFERENCES}

1. Bernstein KT, Marcus JL, Nieri G, et al. Rectal gonorrhea and chlamydia reinfection is associated with increased risk of HIV seroconversion. J Acquir Immune Defic Syndr 2010; 53:537-543. [PubMed: 19935075]

2. Craib KJ, Meddings DR, Strathdee SA, et al. Rectal gonorrhoea as an independent risk factor for HIV infection in a cohort of homosexual men. Genitourin Med 1995; 71:150-154. [PubMed: 7635489]

3. Zetola NM, Bernstein KT, Wong E, et al. Exploring the relationship between sexually transmitted diseases and HIV acquisition by using different study designs. J Acquir Immune Defic Syndr 2009; 50:546-551. [PubMed: 19367993]

4. Annan NT, Sullivan AK, Nori A, et al. Rectal chlamydia-a reservoir of undiagnosed infection in men who have sex with men. Sex Transm Infect 2009; 85:176-179. [PubMed: 19176570]

5. Gunn RA, O'Brien CJ, Lee MA, et al. Gonorrhea screening among men who have sex with men: value of multiple anatomic site testing, San Diego, California, 1997-2003. Sex Transm Dis 2008; 35:845-848. [PubMed: 18607315]

6. Kent CK, Chaw JK, Wong W, et al. Prevalence of rectal, urethral, and pharyngeal chlamydia and gonorrhea detected in 2 clinical settings among men who have sex with men: San Francisco, California, 2003. Clin Infect Dis 2005; 41:67-74. [PubMed: 15937765]

7. Marcus JL, Bernstein KT, Kohn RP, et al. Infections missed by urethral-only screening for chlamydia or gonorrhea detection among men who have sex with men. Sex Transm Dis 2011; 38:922-924. [PubMed: 21934565]

8. Marcus JL, Bernstein KT, Stephens SC, et al. Sentinel surveillance of rectal chlamydia and gonorrhea among males-San Francisco, 2005-2008. Sex Transm Dis 2010; 37:59-61. [PubMed: 20118677]

9. Mimiaga MJ, Mayer KH, Reisner SL, et al. Asymptomatic gonorrhea and chlamydial infections detected by nucleic acid amplification tests among Boston area men who have sex with men. Sex Transm Dis 2008; 35:495-498. [PubMed: 18354345]

10. Young H, Manavi K, McMillan A. Evaluation of ligase chain reaction for the non-cultural detection of rectal and pharyngeal gonorhoea in men who have sex with men. Sex Transm Infect 2003; 79:484-486. [PubMed: 14663126]

11. Workowski KA, Berman S. Sexually transmitted diseases treatment guidelines, 2010. MMWR Recomm Rep 2010; 59:1-110. 
12. Hoover KW, Butler M, Workowski K, et al. STD screening of HIV-infected MSM in HIV clinics. Sex Transm Dis 2010; 37:771-776. [PubMed: 20585275]

13. HIV Epidemiology Section, San Francisco Department of Public Health. HIV/AIDS Epidemiology Annual Report San Francisco, CA: San Francisco Department of Public Health, 2011.

14. Althaus CL, Heijne JCM, Low N. Towards more robust estimates of the transmissibility of Chlamydia trachomatis. Sex Transm Dis 2012; 39:402-404. [PubMed: 22504608]

15. Althaus CL, Heijne JCM, Roellin A, et al. Transmission dynamics of Chlamydia trachomatis affect the impact of screening programmes. Epidemics 2010; 2:123-131. [PubMed: 21352783]

16. Schachter J, Moncada J, Liska S, et al. Nucleic acid amplification tests in the diagnosis of chlamydial and gonococcal infections of the oropharynx and rectum in men who have sex with men. Sex Transm Dis 2008; 35:637-642. [PubMed: 18520976]

17. Turner KME, Adams EJ, LaMontagne DS, et al. Modelling the effectiveness of chlamydia screening in England. Sex Transm Infect 2006; 82:496-502. [PubMed: 17151036]

18. Begley CE, McGill L, Smith PB. The incremental cost of screening, diagnosis, and treatment of gonorrhea and chlamydia in a family planning clinic. Sex Transm Dis 1989; 16:63-67. [PubMed: 2500721]

19. Desai K, Sansom SL, Ackers ML, et al. Modeling the impact of HIV chemoprophylaxis strategies among men who have sex with men in the United States: HIV infections prevented and costeffectiveness. AIDS 2008; 22:1829-1839. [PubMed: 18753932]

20. Hutchinson AB, Farnham PG, Dean HD, et al. The economic burden of HIV in the United States in the era of highly active antiretroviral therapy: Evidence of continuing racial and ethnic differences. J Acquir Immune Defic Syndr 2006; 43:451-457. [PubMed: 16980906]

21. Schackman BR, Gebo KA, Walensky RP, et al. The lifetime cost of current human immunodeficiency virus care in the United States. Med Care 2006; 44:990-997. [PubMed: 17063130]

22. Steece R. Determination of laboratory costs: Or what does it cost to run a nucleic acid amplification test (NAAT).. Presented at: 2008 American Society for Microbiology 108th General Meeting; Boston. 2008.

23. Thompson Healthcare. 2008 Red Book: Pharmacy's Fundamental Reference Montvale, NJ: Thompson Healthcare, 2008.

24. Grosse SD. Assessing cost-effectiveness in healthcare: history of the $\$ 50,000$ per QALY threshold. Expert Rev Pharmacoecon Outcomes Res 2008; 8:165-178. [PubMed: 20528406]

25. Tengs TO. Cost-effectiveness versus cost-utility analysis of interventions for cancer: Does adjusting for health-related quality of life really matter? Value Health 2004; 7:70-78. [PubMed: 14720132]

26. Hu D, Hook EW 3rd, Goldie SJ. Screening for Chlamydia trachomatis in women 15 to 29 years of age: A cost-effectiveness analysis. Ann Intern Med 2004; 141:501-513. [PubMed: 15466767]

27. Gift TL, Gaydos CA, Kent CK, et al. The program cost and cost-effectiveness of screening men for chlamydia to prevent pelvic inflammatory disease in women. Sex Transm Dis 2008; 35:S66-S75. [PubMed: 18830137]

28. Maciosek MV, Edwards NM, Coffield AB, et al. Priorities among effective clinical preventive services: Results of a systematic review and analysis. Am J Prev Med 2006; 31:52-61. [PubMed: 16777543]

29. Cohen CR, Plummer FA, Mugo N, et al. Increased interleukin-10 in the endocervical secretions of women with non-ulcerative sexually transmitted diseases: A mechanism for enhanced HIV-1 transmission? AIDS 1999; 13:327-332. [PubMed: 10199222]

30. Cohen MS, Hoffman IF, Royce RA, et al. Reduction of concentration of HIV-1 in semen after treatment of urethritis: Implications for prevention of sexual transmission of HIV-1. Lancet 1997; 349:1868-1873. [PubMed: 9217758]

31. Fleming DT, Wasserheit JN. From epidemiological synergy to public health policy and practice: The contribution of other sexually transmitted diseases to sexual transmission of HIV infection. Sex Transm Infect 1999; 75:3-17. [PubMed: 10448335] 
32. Hanson J, Posner S, Hassig S, et al. Assessment of sexually transmitted diseases as risk factors for HIV seroconversion in a New Orleans sexually transmitted disease clinic, 1990-1998. Ann Epidemiol 2005; 15:13-20. [PubMed: 15571989]

33. Rottingen JA, Cameron DW, Garnett GP. A systematic review of the epidemiologic interactions between classic sexually transmitted diseases and HIV-How much really is known? Sex Transm Dis 2001; 28:579-597. [PubMed: 11689757]

34. Ward H, Ronn M. Contribution of sexually transmitted infections to the sexual transmission of HIV. Curr Opin HIV AIDS 2010; 5:305-310. [PubMed: 20543605]

35. Centers for Disease Control and Prevention. High-Impact HIV Prevention. CDC's Approach to Reducing HIV Infections in the United States Atlanta, GA: CDC, 2011. 


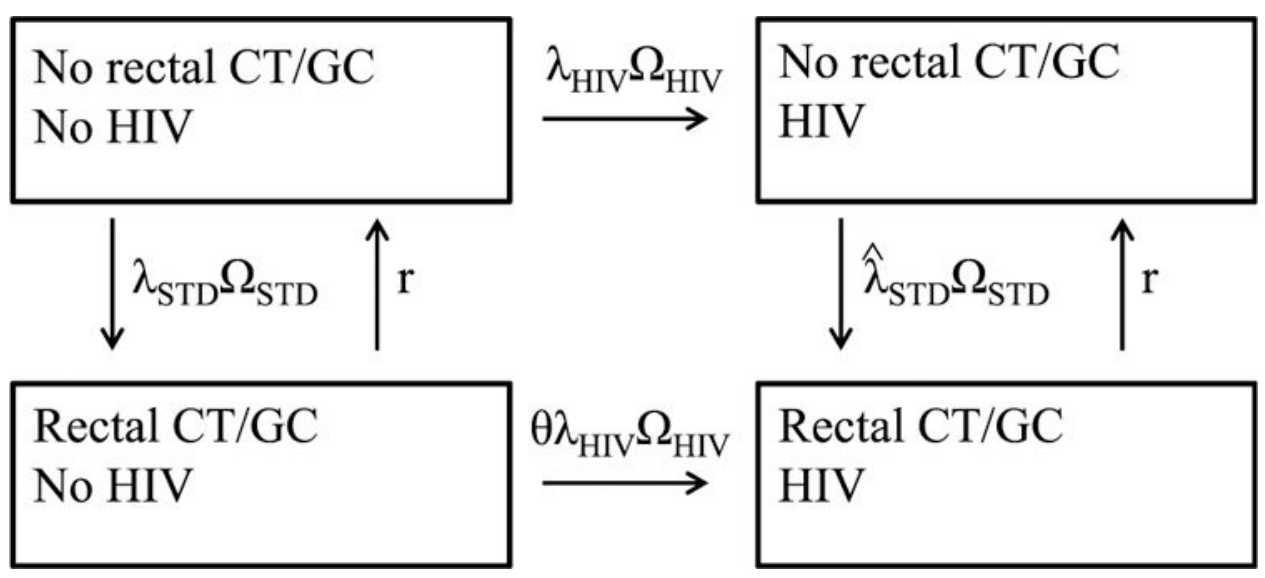

Figure 1.

Illustration of the model. The 4 health states are mutually exclusive, and transition occurs when rectal $C T / G C$ infection is acquired, when rectal $C T / G C$ infection is cleared, or when HIV is acquired. The HIV incidence rate among those without rectal $C T / G C$ infection is $\lambda_{\mathrm{HIV}} \Omega_{\mathrm{HIV}}$, where $\lambda_{\mathrm{HIV}}$ is the HIV incidence at the onset of the screening program and $\Omega_{\mathrm{HIV}}$ is an adjustment factor to account for changes in the prevalence of HIV in sex partners over time. The rate of HIV incidence among those with rectal $C T / G C$ infection is assumed to be $\theta$ times that of those without rectal $C T / G C$ infection. The incidence rate of rectal $C T / G C$ infection is $\lambda_{\mathrm{STD}} \Omega_{\mathrm{STD}}$ and $\hat{\lambda}_{\mathrm{STD}} \Omega_{\mathrm{STD}}$ among those without and with HIV, respectively, where $\lambda_{\mathrm{STD}}$ and $\hat{\lambda}_{\mathrm{STD}}$ are the incidence rates of rectal $C T / G C$ infection at the onset of the screening program among those without and with HIV, respectively, and $\Omega_{\mathrm{STD}}$ is an adjustment factor to account for changes in the prevalence of $C T$ and $G C$ in sex partners over time as a result of the screening program. The clearance rate of rectal $C T / G C$ infection $(r)$ is a function of 2 factors: duration of infection and screening rates. 


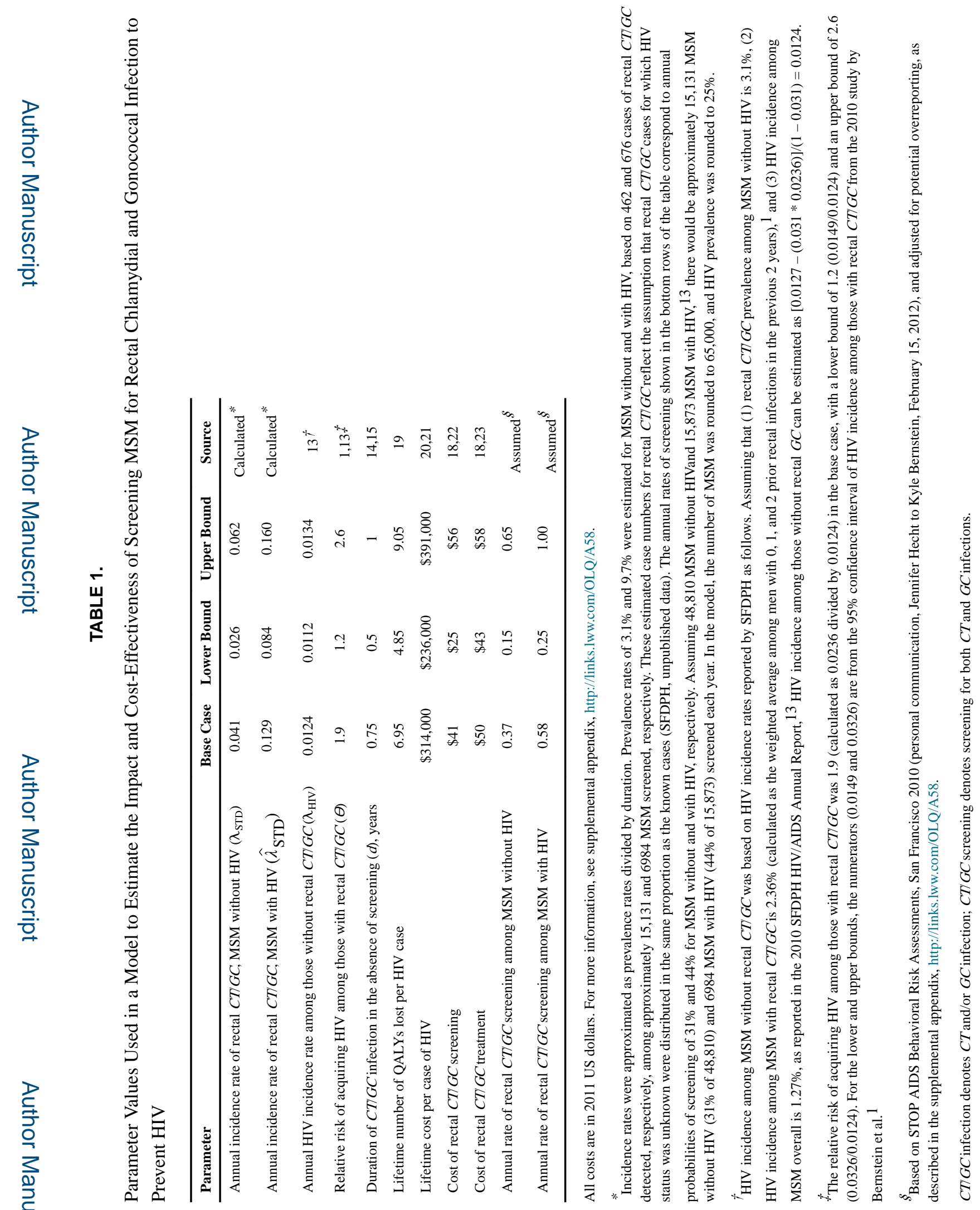




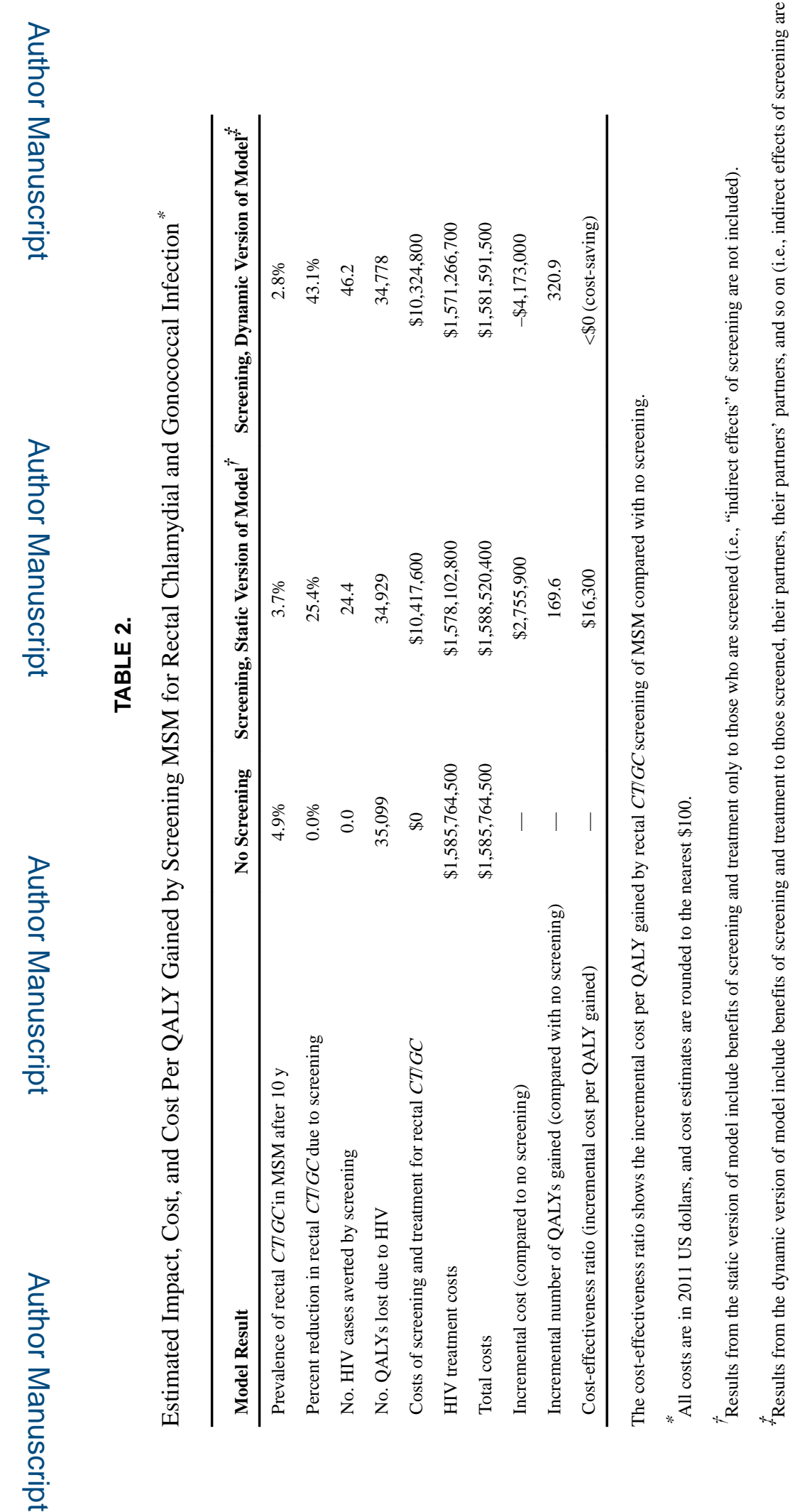

Sex Transm Dis. Author manuscript; available in PMC 2019 September 16. 


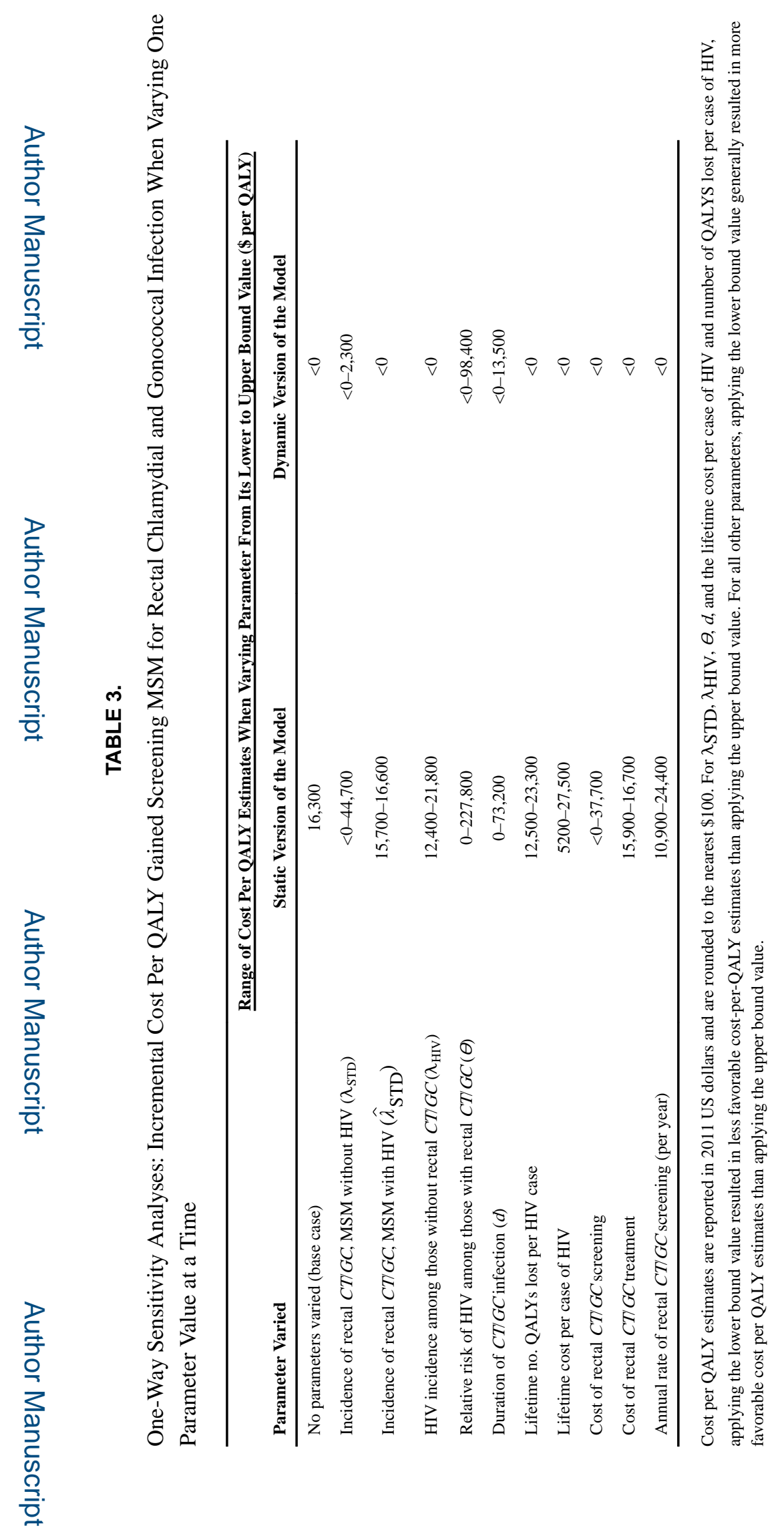

Sex Transm Dis. Author manuscript; available in PMC 2019 September 16. 


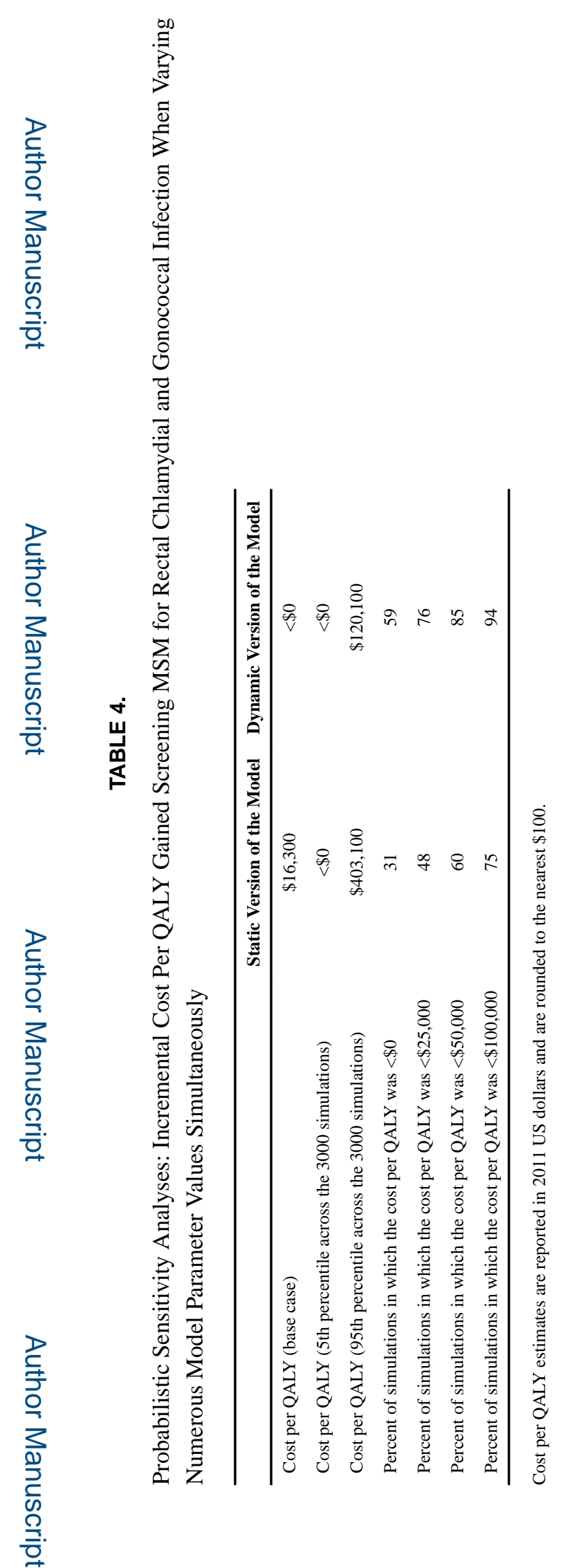

Sex Transm Dis. Author manuscript; available in PMC 2019 September 16. 